\title{
Multifractal Analysis and Micromorphologies of Radiation Synthesized Hydrogel Polymers
}

\author{
Shan Jun, Chen Jie, ${ }^{*}$ Liv Zhanjun, ${ }^{* *, \dagger}$ and Zhang Maosen* \\ College of Chemical Engineering, Hefei University of Technology, Hefei 230009, \\ People's Republic of China \\ * Department of Chemical Physics, University of Science and Technology of China, Hefei 230026, \\ People's Republic of China \\ ** Department of Applied Chemistry, University of Science and Technology of China, Hefei 230026 , \\ People's Republic of China
}

(Received April 4, 1996)

\begin{abstract}
Polyacrylamide (PAM) and poly sodium acrylate (PNaA) homopolymer hydrogels and five kinds of $\mathrm{P}(\mathrm{AM}-\mathrm{NaA})$ copolymer hydrogels with different monomer molar ratios $\mathrm{AM} / \mathrm{NaA}$ were synthesized by ${ }^{60} \mathrm{Co}-\gamma$ ray radiation polymerization in aqueous solutions. The micromorphologies of these hydrogel polymer samples were observed by a scanning electron microscope (SEM). From the interaction between the chain segments of polymers, it is qualitatively expounded that the cross-linking network structure of hydrogel polymers displayed successively the thicker framework network $\rightarrow$ incomplete bubble film network $\rightarrow$ dense network with the increase of $\mathrm{NaA}$ units in the hydrogel polymer chains. The non-homogeneity and complexity of micro network distribution of hydrogel polymers were elucidated by the multifractal theory. The results show that the multifractal spectrum, singular index $\alpha$ and Hausdorff dimension $D_{0}$ can quantitatively characterize the non-homogeneity and the width of network distribution of hydrogel homopolymers and copolymers.
\end{abstract}

KEY WORDS Multifractal Micromorphology / Hydrogel / Radiation Polymerization /

The fractal theory advanced by Mandelbrot has become a kind of science which can describe the laws of many irregular objects in nature. ${ }^{1,2)}$ And multifractal theory can elucidate the fine structure of complex fractals which have non-homogeneity of inter scale..$^{3,4}$

The hydrogel polymer is a kind of intelligent polymer material which can sense sharply the stimulus of circumstance and form the judgment and make the response. ${ }^{5,6}$ It has been applied to the drug release and delivery system and the chemo-mechanical system.

In our previous work, ${ }^{7,8}$ the features of the volume phase transition of several hydrogels in various concentrations of acetone-water solution were studied and their micromorphologies were observed. It was found that with the variance of composition of the hydrogel polymers their micromorphologies displayed both obviously different and interrelated micro network structures. The internal relation between micro structure and property of volume phase transition of the hydrogel polymers has been in progress. Here, the multifractal theory is used to quantitatively characterize the nonhomogeneity and inner fine structure of the micromorphologies of PAM, PNaA, and P(AM-NaA) hydrogel polymers.

\section{EXPERIMENTAL}

\section{Materials}

Acrylamide (AM) and sodium acrylate (NaA) monomers and $N, N^{\prime}$-methylenebisacrylamide (MBA) crosslinking agent were employed. AM was recrystallized twice in acetone solvent before use. $\mathrm{NaA}$ monomer was prepared from acrylic acid (AA), which was purified by reduced pressure distillation. MBA was used without further purification. AM, AA, and MBA were all purchased from Shanghai Chem. Co.

\section{Synthesis of Hydrogel Polymers by Radiation Polymeriza- tion}

At first, certain amount of AA was completely neutralized by $0.1000 \mathrm{~mol} \mathrm{~L}^{-1} \mathrm{NaOH}$ aqueous solution. Then, $\mathrm{NaA}$ aqueous solution with definite concentration was obtained whose $\mathrm{pH}$ value was about 7.5. AM and $\mathrm{MaA}$ aqueous solutions and five kinds of $\mathrm{AM}$ and $\mathrm{NaA}$ mixed aqueous solutions with different monomer molar ratios $\mathrm{AM} / \mathrm{NaA}$ were made up respectively, keeping the total molar quantity of the two monomers constant and adding an appropriate amount of crosslinker MBA in above aqueous solutions (the molecular fraction $f_{\mathrm{MBA}}=$ 0.012 ).

Each of the above solutions was poured into an irradiation bottle into which many clean PTFE tubes with internal diameter $1.64 \mathrm{~mm}$ were vertically put. All the irradiation bottles with solutions were flushed with nitrogen for about $30 \mathrm{~min}$, and then were sealed. The absorbed dose and irradiation time were so selected that conversion of two monomers could reach near $100 \%$. The radiation polymerization was carried out at room temperature. The hydrogels removed from every PTFE tube are colorless and transparent, and have some strength and elasticity. Table I lists the data for the preparation of hydrogels. On the basis of the reactivity ratios of two monomers ${ }^{9} \gamma_{\mathrm{AM}}=0.99-1.10$ and $\gamma_{\mathrm{NaA}}=$ $0.13-0.45$, it can be foreseen that the distribution of two monomer units in the chain segments of $\mathrm{P}(\mathrm{AM}-\mathrm{NaA})$ copolymers is very homogeneous and the monomer unit molar ratio in the copolymer is almost the same as that in the corresponding aqueous solution.

\footnotetext{
† Author to whom correspondence is addressed (e-mail: zjliu@ach.ustc.ac.cn).
} 
Table I. Formulation and absorbed dose for the preparation of hydrogel samples by ${ }^{60} \mathrm{Co}-\gamma$ ray radiation polymerization ${ }^{\mathrm{a}}$

\begin{tabular}{lccccccc}
\hline Sample No. & $\mathrm{I}$ & $\mathrm{II}_{1}$ & $\mathrm{II}_{2}$ & $\mathrm{II}_{3}$ & $\mathrm{II}_{4}$ & $\mathrm{II}_{5}$ & $\mathrm{III}$ \\
\hline $\mathrm{AM} / \mathrm{NaA}$ & $10 / 0$ & $9.5 / 0.5$ & $9.0 / 1.0$ & $8.0 / 2.0$ & $6.0 / 4.0$ & $4.0 / 6.0$ & $0 / 10$ \\
$f_{\mathrm{MBA}}$ & 0.012 & 0.012 & 0.012 & 0.012 & 0.012 & 0.012 & 0.012 \\
$D / \mathrm{Gy}$ & 800 & 800 & 800 & 800 & 800 & 800 & 1000 \\
\hline
\end{tabular}

${ }^{\text {a }} \mathrm{AM} / \mathrm{NaA}$, molar ratio of the two monomers; $D$, absorbed dose.

\section{Measurements of Micromorphologies of Hydrogel Poly- mers}

SEM micrographs of hydrogel polymers were taken on a X-650 scanning electron microscope. The colorless and transparent hydrogels were dehydrated for 48 hours at room temperature in acetone solvent, and became white and hard bars. These bars were dried under infrared lamp to remove the residual adsorbed acetone. By this way, the dry hydrogel samples were obtained. Then, the samples were fractured carefully to reveal the fresh cross sections and mounted on a stub using silver paint. Subsequently the fresh cross sections of samples were coated with gold on a Eiko IB-3 ion coater. The typical magnification employed was $10.0 \mathrm{k}$.

\section{CALCULATION METHODS OF MULTIFRACTAL}

The $P_{i}$ is defined as the density distribution function in the $i$ th interval, $P_{i}(\varepsilon)-\varepsilon^{\alpha}$, then

$$
\alpha=\lim _{\varepsilon \rightarrow 0} \frac{\ln P_{i}(\varepsilon)}{\ln \varepsilon}
$$

where $\varepsilon$ is the measurement unit which is far less than one. The non-integer index $\alpha$ is called singular index. The numbers of the small intervals which have the same $\alpha$ values are called $N_{\alpha}$, then

$$
N_{\alpha}(\varepsilon) \propto \varepsilon^{-f(\alpha)}
$$

Hence, the $f(\alpha)$ indicates the dimension of the daughter sets which have the same $\alpha$ values. However, the distribution of $P_{i}$ is generally unknown. So the feature of the distribution is often obtained by means of the "moment" of statistical physics. Thus, $\chi_{q}(\varepsilon)$, which is the sum of weighted $P_{i}$, is defined as the following equations:

$$
\begin{aligned}
& \chi_{q}(\varepsilon)=\sum P_{i}^{q}=\varepsilon^{\tau(q)} \\
& \tau(q)=\lim _{\varepsilon \rightarrow 0} \frac{\ln \chi(q)}{\ln \varepsilon}
\end{aligned}
$$

Correspondingly, the weighted generalized fractal dimension $D_{q}$ is

$$
D_{q}=\frac{1}{q-1} \lim _{\varepsilon \rightarrow 0} \frac{\ln \chi_{q}(\varepsilon)}{\ln \varepsilon}=\frac{\tau(q)}{q-1}
$$

It can be seen from eq 5 that $\tau(0)=-D_{0}$ when $q=0$ and $\tau(1) \equiv 0$,

$$
\chi_{1}(\varepsilon)=\sum_{i} P_{i} \equiv 1 \quad \text { when } \quad q=1 .
$$

$D_{0}$ is just Hausdorff dimension or fractal dimension.

Equations $1-2$ are substituted into eq 3, then

$$
\begin{aligned}
& \chi_{q}(\varepsilon) \propto N(\alpha, \varepsilon) P_{i}^{q} \propto \varepsilon^{-f(\alpha)+\alpha(q)} \propto \varepsilon^{\tau(q)} \\
& \tau(q)=\alpha q-f(\alpha)
\end{aligned}
$$

Generally, $\tau(q)$ is called the mass index. Equations 5 and 7 are combined into eq 8 :

$$
\alpha=\frac{\mathrm{d} \tau(q)}{\mathrm{d} q}
$$

The above formulas constitute the heart of the multifractal theory.

\section{RESULTS AND DISCUSSION}

\section{Effect of Composition of Hydrogel Samples on the Features} of Their Micromorphologies

SEM micrographs of hydrogel samples are respectively shown in Figure 1 . It is evident that their micromorphologies differ greatly.

For homopolymer sample I(PAM), its micromorphology shows clearly the cross-linked network structure. The interaction among the chain segments is mainly the attraction of hydrogen bond developed between amide groups as side groups in chain of PAM. As a result, many strands of chain segments of PAM tend to form some bundles of thicker network frameworks. The network distribution has non-homogeneity.

The micromorphology of every $\mathrm{P}(\mathrm{AM}-\mathrm{NaA})$ copolymer sample differs from that of the homopolymer sample I(PAM). Furthermore, the differences among the micromorphologies of five kinds of $\mathrm{P}(\mathrm{AM}-\mathrm{NaA})$ copolymer samples are obvious. Two kinds of side groupsamide groups and carboxyl anions exist in the chain segments of $\mathrm{P}(\mathrm{AM}-\mathrm{NaA})$ copolymers. Their micromorphologies mainly depend on two kinds of opposite forces among the chain segments of copolymers-the hydrogen bond attraction between amide groups (including $-\mathrm{N}-\mathrm{H} \cdots \mathrm{O}$ and $-\mathrm{N}-\mathrm{H} \cdots \mathrm{N}$ hydrogen bond) and the electrostatic repulsion among carboxyl anions. Because the existence of electrostatic repulsion can weaken the attraction of hydrogen bond, the tendency in which many strands of chain segments are inclined to form the thicker network frameworks by hydrogen bond attraction is weakened, whereas many strands of chain segments tend to develop some cambers which have definite thickness. For copolymer sample $\mathrm{II}_{1}$, the content of $\mathrm{NaA}$ unit is far lower than that of AM unit and the distribution of the carboxyl anions in chain segments is sparse. Its micromorphology begins to show some difference compared with that of homopolymer sample I(PAM), that is, the above mentioned cambers appear in its network structure. But, there is some similarity between their micromorphologies.

With the increase of the content of carboxyl anions distributed in chain segments of copolymers, the attraction of hydrogen bond is quitely weakened, so the above cambers develop the bubble films. Hence, the micromorphologies of $\mathrm{II}_{2}, \mathrm{II}_{3}$, and $\mathrm{II}_{4}$ display the stereoscopic incomplete bubble film network structure, and all of incomplete bubbles communicate with each other. It is still attributed to the interaction between two kinds of the opposite forces among the chain segments of copolymers. 


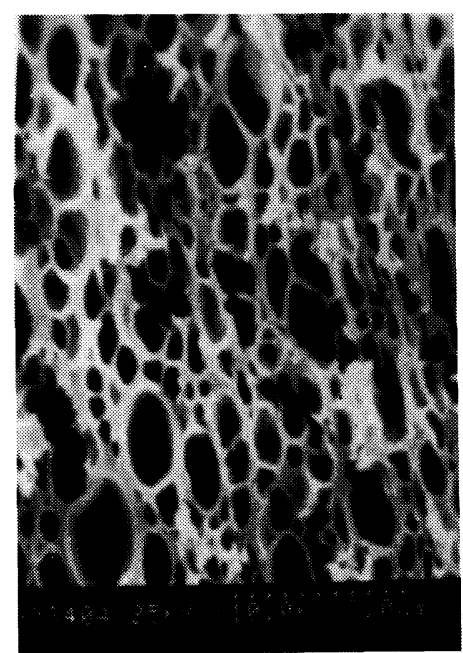

(I)

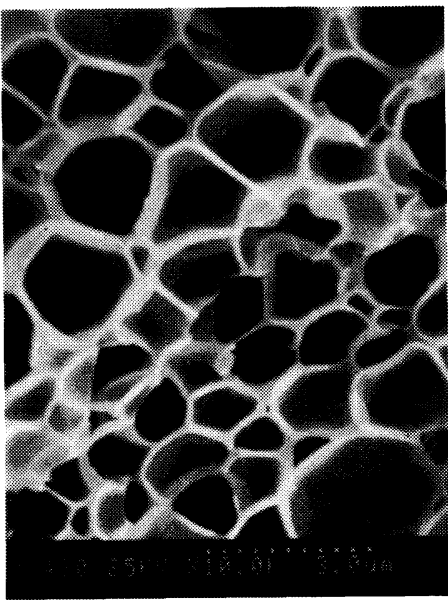

$\left(\mathrm{II}_{3}\right)$

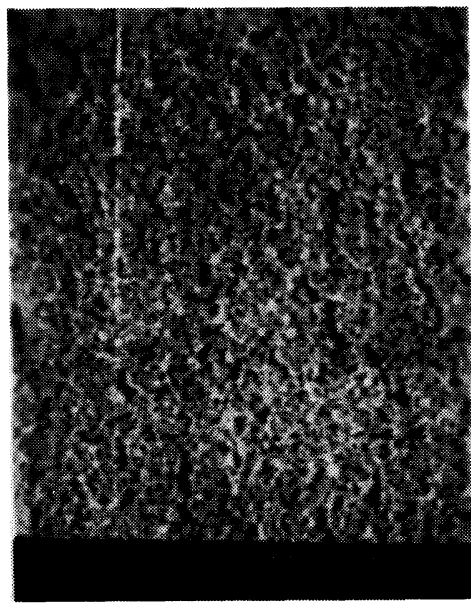

(III)

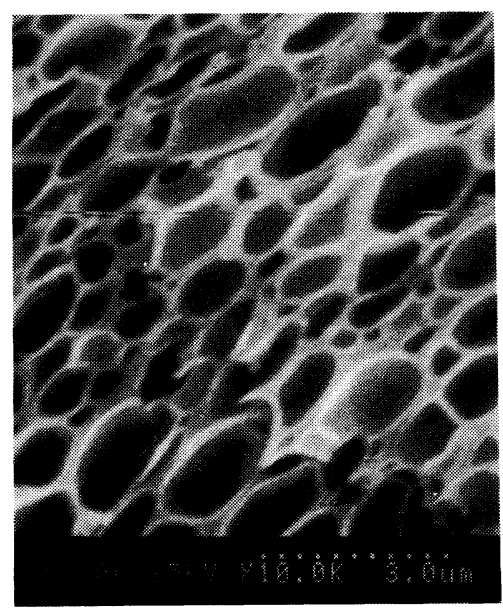

$\left(\mathrm{II}_{1}\right)$

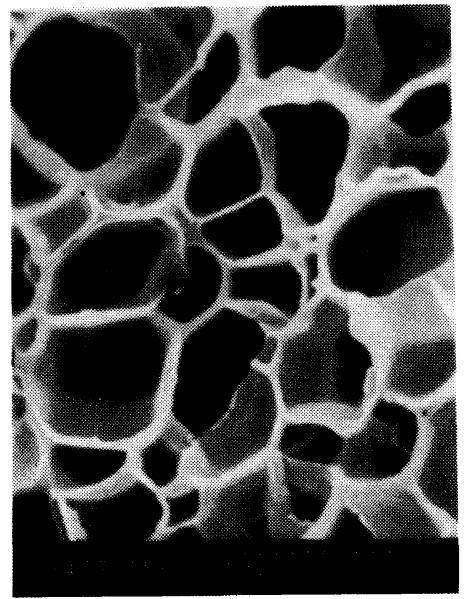

$\left(\mathrm{II}_{4}\right)$

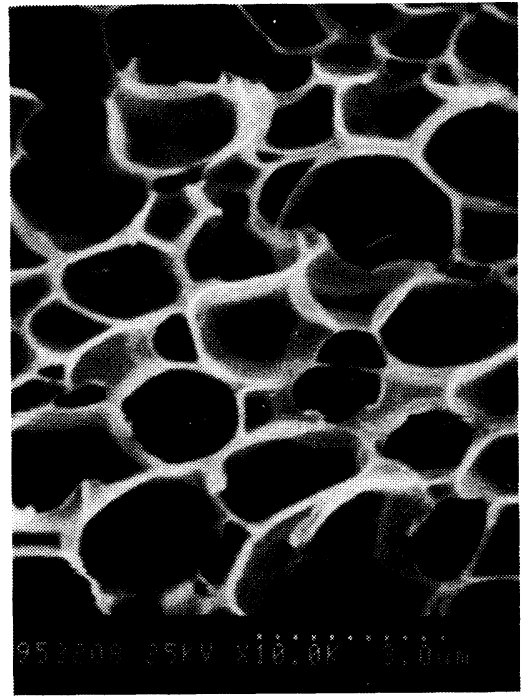

$\left(\mathrm{II}_{2}\right)$

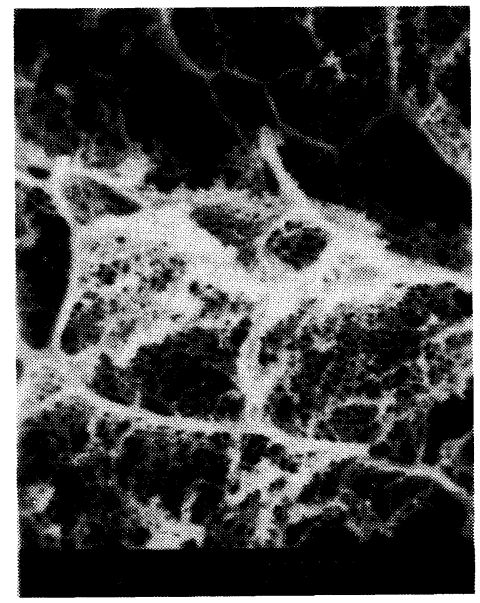

$\left(\mathrm{II}_{5}\right)$

Figure 1. SEM micrographs of hydrogel polymers.

However, for copolymer sample $\mathrm{II}_{5}$, when the content of carboxyl anions increases up to a certain extent, the electrostatic repulsion among the chain segments occupies the leading position. Consequently, the incomplete bubble film network structure mentioned above disappears. The micromorphology of copolymer sample $\mathrm{II}_{5}$ shows the dense network structure which looks like being woven out of thinner network frameworks.
Homopolymer sample III( $\mathrm{PNaA})$ can be taken as an extreme example in which the side groups in the chain segments are all carboxyl anions. Its micromorphology shows much denser network structure than that of any other sample.

Thus, it can be seen that the content of carboxyl anions affects greatly the micromorphologies of hydrogel polymers. 


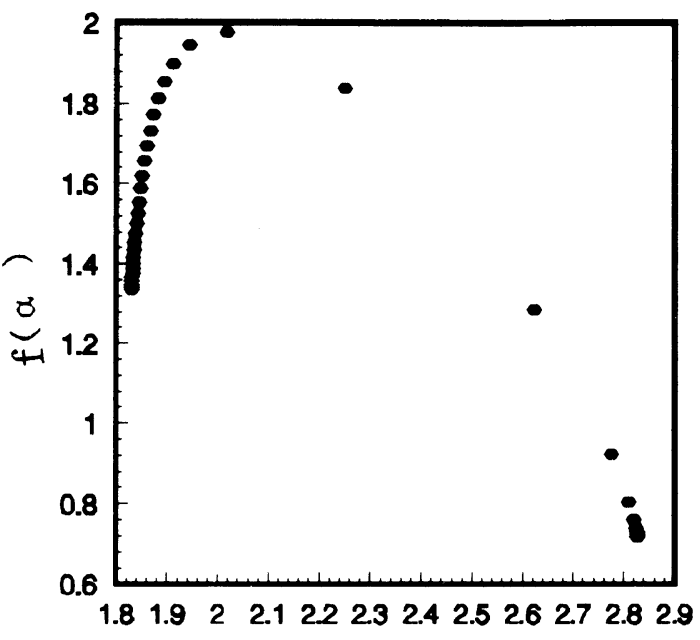

$a$

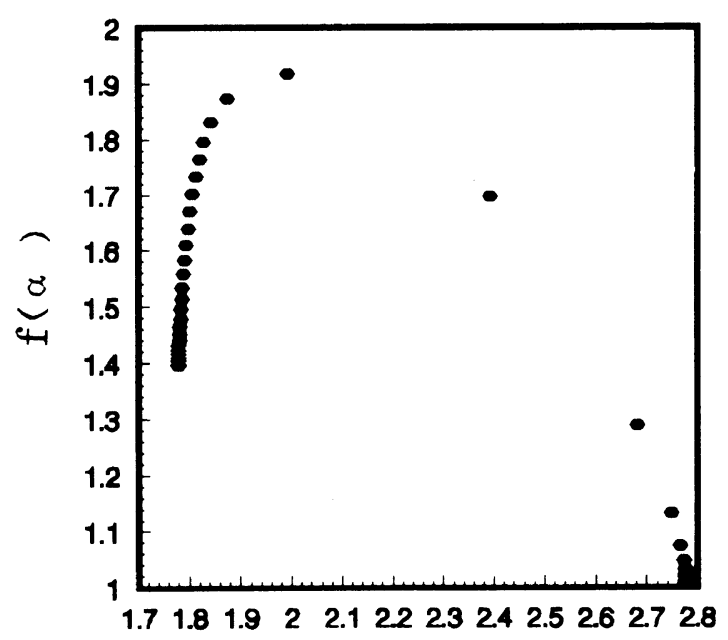

$\alpha$

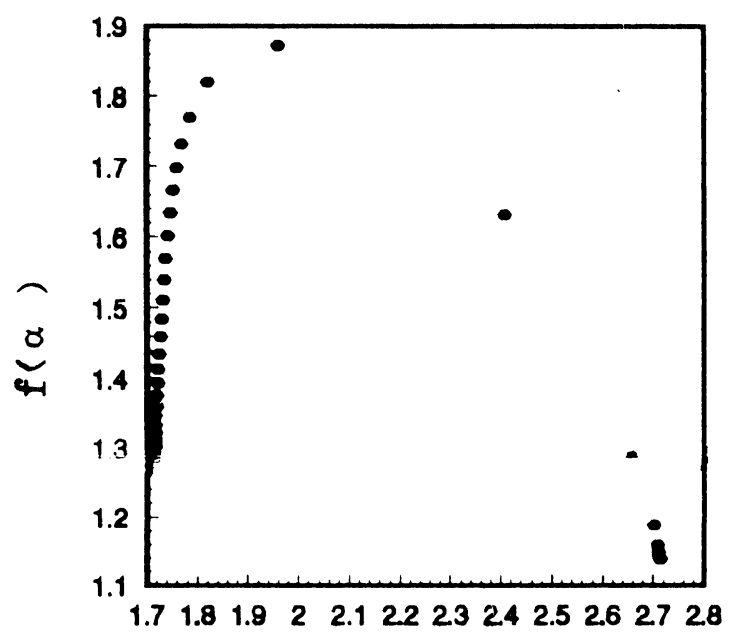

$\alpha$

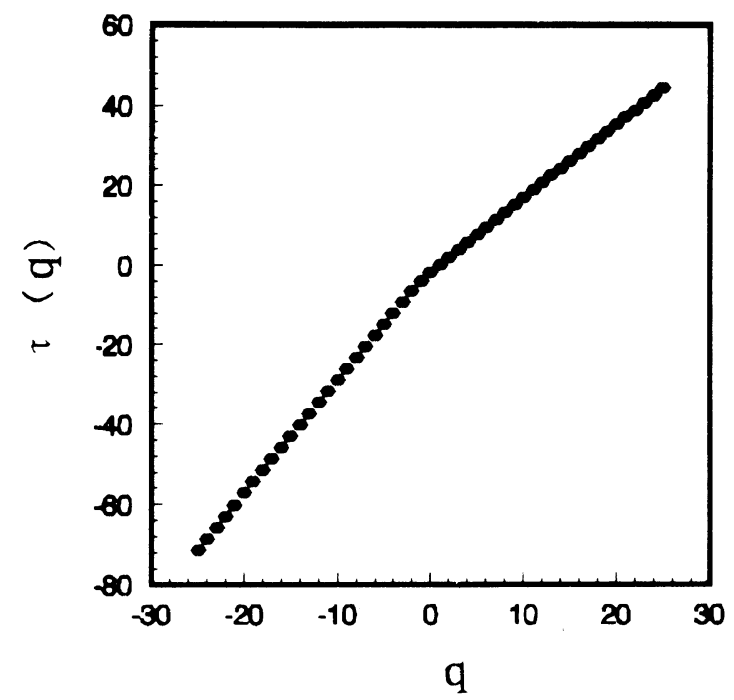

(I)

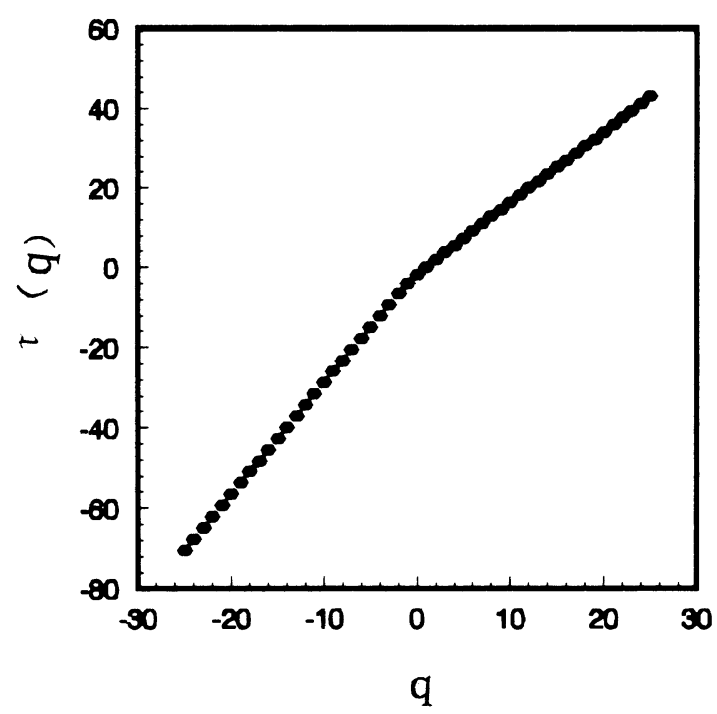

$\left(\mathrm{II}_{1}\right)$

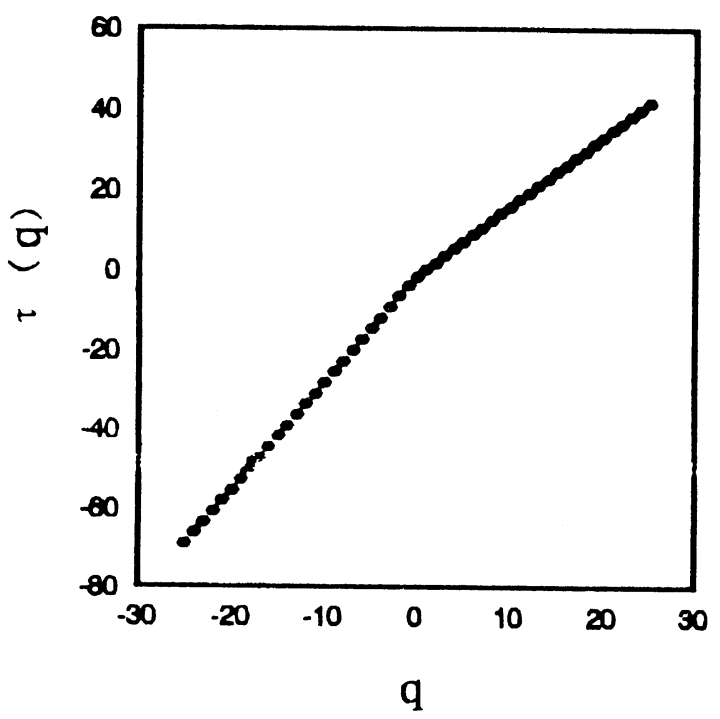

$\left(\mathrm{II}_{2}\right)$ 


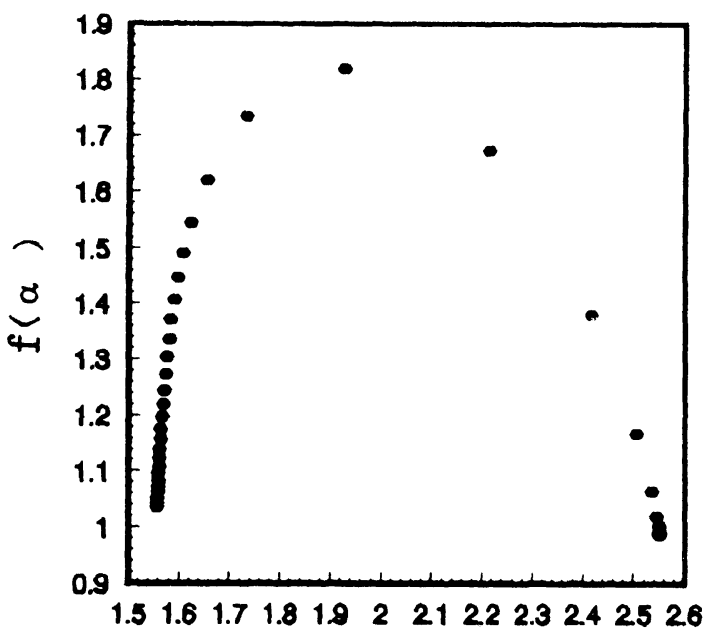

$a$

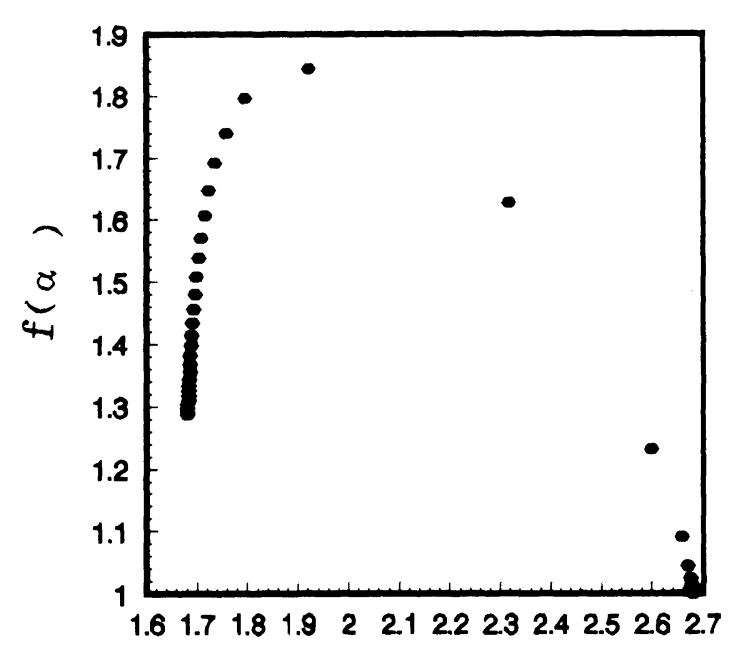

$\alpha$

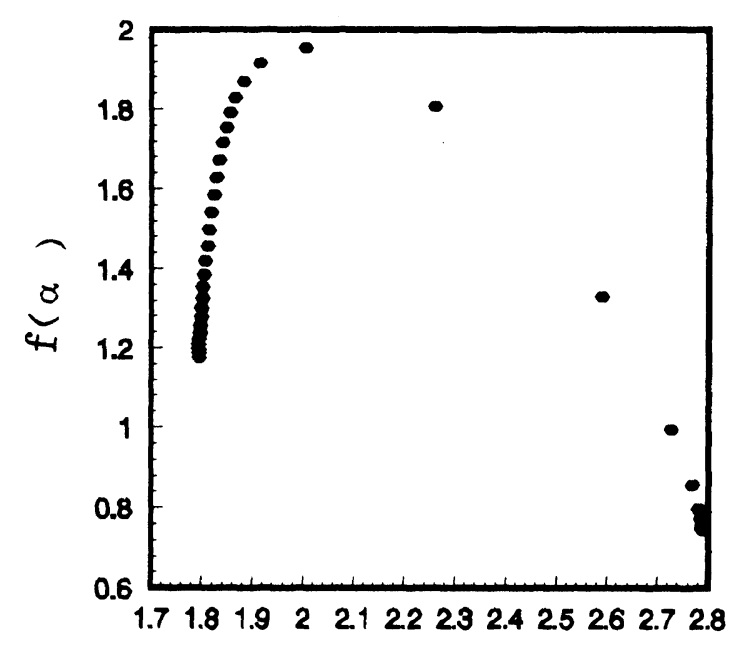

$\alpha$

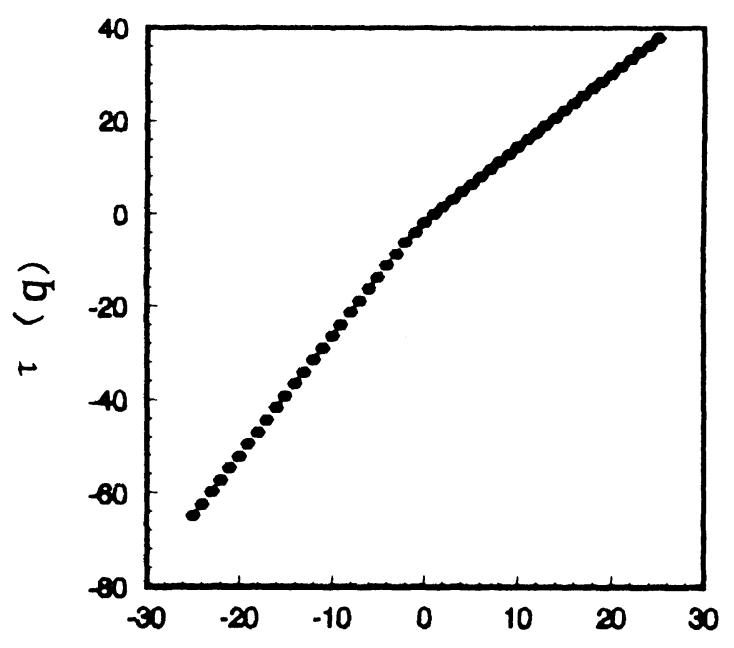

q

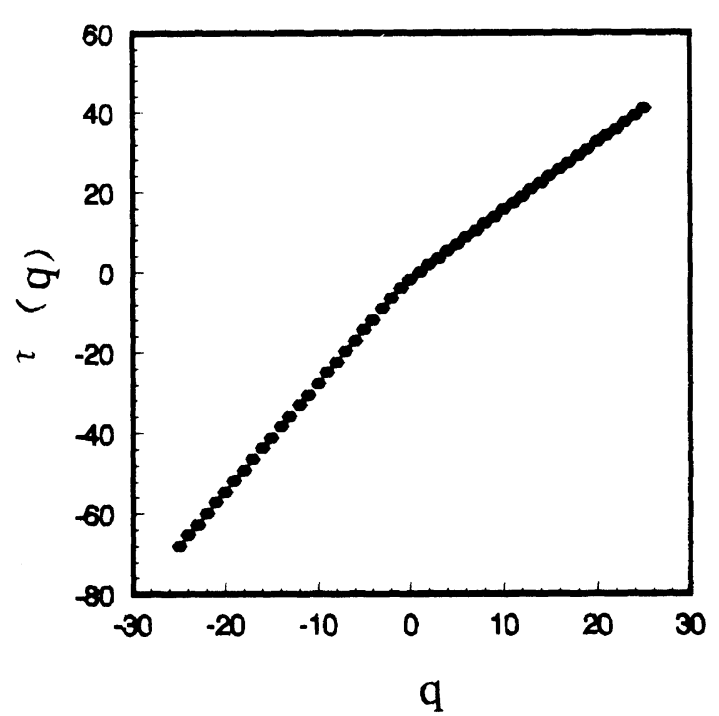

$\left(\mathrm{II}_{4}\right)$

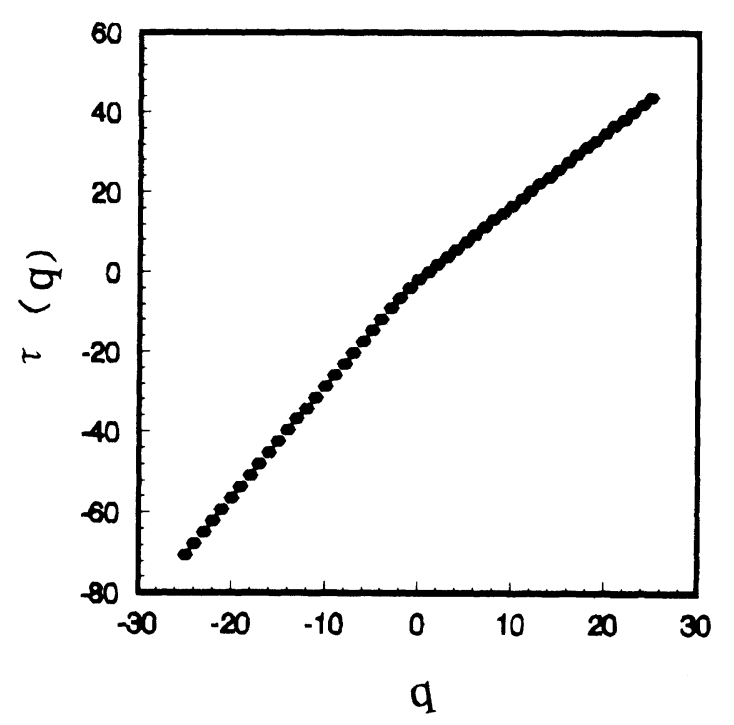

$\left(\mathrm{II}_{5}\right)$

Figures $2\left(\mathrm{II}_{3}\right) \sim\left(\mathrm{II}_{5}\right)$

(for the legend of Figure 2, see page 891) 


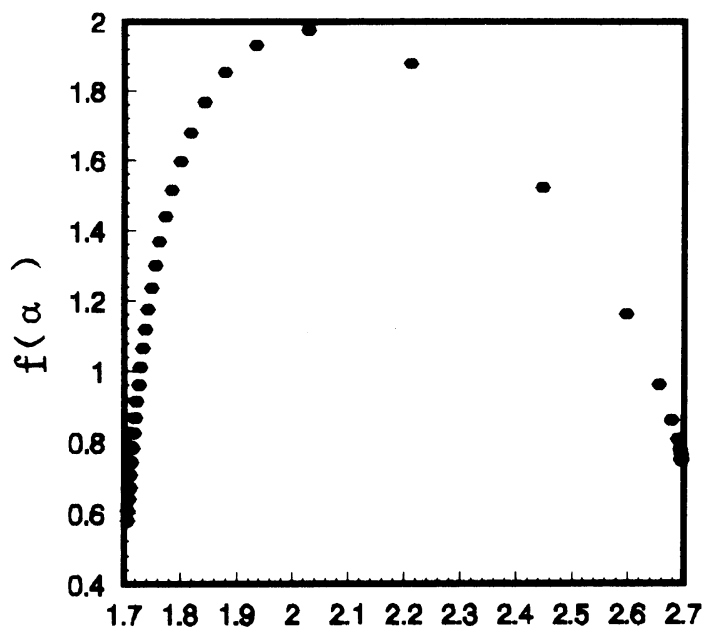

$\alpha$

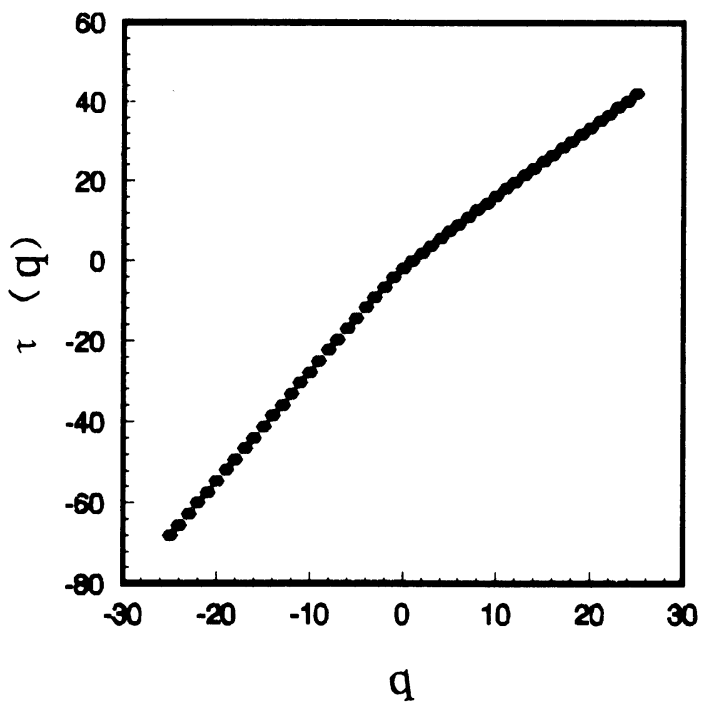

q

(III)

Figures 2 (III)

Figure 2. Multifractal spectra $f(\alpha)-\alpha$ and $\tau(q)-q$ curves of hydrogel polymers.

Table II. The data of multifractal analysis of micromorphologies of polymer samples

\begin{tabular}{cccccc}
\hline Sample No. & $\Delta \alpha$ & $f\left(\alpha_{\min }\right)$ & $f\left(\alpha_{\max }\right)$ & $D_{0}=f(\alpha)_{q=0}$ & $D_{1}=f(\alpha)_{q=1}=\alpha_{q=1}$ \\
\hline I & 0.9974 & 1.333 & 0.715 & 1.975 & 1.944 \\
II $_{1}$ & 0.9986 & 1.395 & 1.012 & 1.918 & 1.873 \\
II $_{2}$ & 0.9976 & 1.282 & 1.138 & 1.872 & 1.819 \\
$\mathrm{II}_{3}$ & 0.9948 & 1.035 & 0.987 & 1.819 & 1.734 \\
$\mathrm{II}_{4}$ & 0.9981 & 1.287 & 1.000 & 1.844 & 1.795 \\
$\mathrm{II}_{5}$ & 0.9964 & 1.176 & 0.745 & 1.952 & 1.916 \\
$\mathrm{III}$ & 0.9895 & 0.580 & 0.745 & 1.976 & 1.933
\end{tabular}

Multifractal Analysis of Micromorphologies of Hydrogel Polymers

Figure 2 shows respectively the multifractal spectra $f(\alpha)-\alpha$ and the $\tau(q)-q$ curves of hydrogel polymers.

All of the distribution curves of $f(\alpha)$ versus $\alpha$ are bell in shape. The distribution width which is expressed with $\Delta \alpha=\alpha_{\max }-\alpha_{\min }$ can indicate the non-homogeneity of micro network distribution of polymer samples. It can be seen that the micro network distribution of copolymers is wider than that of homopolymers; for two homopolymers, the micro network distribution of sample $\mathrm{III}(\mathrm{PNaA})$ is narrower than that of sample I(PAM).

Here, singular index $\alpha$ can describe the density distribution of micro network framework within every small interval on the micro morphology of the polymer sample. By comparison $f\left(\alpha_{\min }\right)$ with $f\left(\alpha_{\max }\right)$ of each polymer sample, the fact that the $f\left(\alpha_{\min }\right)$ value is bigger than the $f\left(\alpha_{\max }\right)$ value (except homopolymer sample III) shows that the dimension corresponding to the interval of the greatest density distribution of the network framework of polymer sample is larger than that corresponding to the interval of the least density distribution of the network framework of the sample. Yet, for homopolymer sample III(PNaA), the $f\left(\alpha_{\text {min }}\right)$ value is lower than the $f\left(\alpha_{\max }\right)$ value.

The $f(\alpha)$ at $q=0$ is Hausdorff dimension $D_{0}$. It represents the whole property of micromorphology of the polymer sample. From the fact that $D_{0}$ values of two homopolymer samples I(PAM) and III(PNaA) are larger than those of five copolymer samples and from the physical meaning of $f(\alpha)$. It can be deduced that the micro network distribution of each homopolymer is denser and more homogeneous than those of copolymers.

Of the Hausdorff dimension $D_{0}$ values of five copolymer samples, the $D_{0}$ values of sample $\mathrm{II}_{1}$ and $\mathrm{II}_{5}$ are larger than those of the rest. The result indicates that the micro network structure of the former is denser and more homogeneous than that of the latter. Furthermore, the $D_{0}$ values of the copolymer samples $\mathrm{II}_{1}$ and $\mathrm{II}_{5}$ are near the ones of the two homopolymer samples I(PMA) and III(PNaA). This further shows that the micromorphologies of $\mathrm{II}_{1}$ and $\mathrm{II}_{5}$ is located at two sorts of the transition states just between the incomplete bubble film network structure of copolymer samples $\mathrm{II}_{2}, \mathrm{II}_{3}$, and $\mathrm{II}_{4}$ and the micromorphologies of two homopolymer samples I(PAM) and III(PNaA) (see Scheme 1 below).

thicker framework network transition state one

(I)

$$
\left(\mathrm{II}_{1}\right)
$$

incomplete bubble film network transition state two denser network

$$
\left(\mathrm{II}_{2}, \mathrm{II}_{3} \mathrm{II}_{4}\right) \quad\left(\mathrm{II}_{5}\right)
$$

Scheme 1. Mechanics of micro morphologes of polymers with content of carboxyl anions in chain segments.

For the rest copolymer samples $\mathrm{II}_{2}, \mathrm{II}_{3}$ and $\mathrm{II}_{4}$, their micromorphologies display non-homogeneity and rough cross sections because of the appearance of the incomplete buttle film network structure.

Besides, the same results as above mentioned ones are still got from the analysis of the information dimension 
$D_{1}$ values of polymer samples which are equal to the $f(\alpha)$ when $q=1$ (see Table II).

The Hausdorff dimension $D_{0}$ values are also obtained from the $\tau(q)-q$ curves of the polymer samples. It can be seen from eq 5 and eq 7 that $D_{0}=-\tau(0)$ and $\tau(0)=$ $-f(\alpha)_{q=0}$ when $q=0$. Thus, $D_{0}=f(\alpha)_{q=0}=-\tau(0)$.

\section{CONCLUSIONS}

The content of carboxyl anions can greatly influence the micromorphologies of hydrogel copolymers. The reason is qualitatively elucidated from the two kinds of opposite force - the hydrogen bond attraction and the electrostatic repulsion which exist in the chain segments of hydrogel copolymers.

The tentative application of the multifractal theory in the micromorphologies of the hydrogel polymers can reveal their inner fine structure in different levels and quantitatively characterize the width and non-homogeneity of micro network distribution.

\section{REFERENCES}

1. B. B. Mandelbrot, "Fractals: Form, Chance and Dimension," W. H. Freeman, San Francisco, Cal., 1977.

2. B. B. Mandelbrot, "The Fractal Geometry of Nature," W. H. Freeman, San Francisco, Cal., 1982.

3. T. C. Halsey, Phys. Rev. A., 33, 1141 (1986).

4. B. B. Mandelbrot, Physica, A, 163, 306 (1990).

5. T. Tanaka, I. Nishio, and S. T. Sun, Science, 218, 467 (1982)

6. D. Nicoli, C. Young, and T. Tanaka, Macromolecules, 16, 887 (1983).

7. J. Shan and Z. J. Liu, Radiat. Phys. Chem., 42, 955 (1993).

8. J. Shan and Z. J. Liu, Chem. J. Chin. Univ., 16, 1467 (1995).

9. H. S. Xu, Z. C. Zhang, and W. J. Liu, Radiat. Res. \& Radiat. Proc., 3, 19 (1985). 